\title{
Texture analysis of intermediate-advanced hepatocellular carcinoma: prognosis and patients' selection of transcatheter arterial chemoembolization and sorafenib
}

\author{
Sirui Fu${ }^{1}$, Shuting Chen ${ }^{2,3}$, Changhong Liang ${ }^{3}$, Zaiyi $\mathrm{Liu}^{3}$, Yanjie Zhu ${ }^{4}$, Yong $\mathbf{L i}^{1}$, \\ Ligong Lu ${ }^{1}$ \\ ${ }^{1}$ Department of Interventional Oncology, Guangdong Provincial Cardiovascular Institute, Guangdong General Hospital, \\ Guangdong Academy of Medical Sciences, Guangzhou, China \\ ${ }^{2}$ Southern Medical University, Guangzhou, China \\ ${ }^{3}$ Department of Radiology, Guangdong General Hospital, Guangdong Academy of Medical Sciences, Guangzhou, China \\ ${ }^{4}$ Shenzhen Institutes of Advanced Technology, Shenzhen, China \\ Correspondence to: Ligong Lu, email: IIg0902@sina.com
}

Keywords: hepatocellular carcinoma, texture analysis, sorafenib, transcatheter arterial chemoembolization

Received: July 20, $2016 \quad$ Accepted: November 14, $2016 \quad$ Published: November 29, 2016

Copyright: Fu et al. This is an open-access article distributed under the terms of the Creative Commons Attribution License

(CC-BY), which permits unrestricted use, distribution, and reproduction in any medium, provided the original author and source are credited.

\section{ABSTRACT}

Transcatheter arterial chemoembolization (TACE) and sorafenib combination treatment for unselected hepatocellular carcinoma (HCC) is controversial. We explored the potential of texture analysis for appropriate patient selection. There were 261 HCCs included (TACE group: $n=197$; TACE plus sorafenib (TACE+Sorafenib) group $n=64)$. We applied a Gabor filter and wavelet transform with 3 band-width responses (filter $0,1.0$, and 1.5) to portal-phase computed tomography (CT) images of the TACE group. Twenty-one textural parameters per filter were extracted from the region of interests delineated around tumor outline. After testing survival correlations, the TACE group was subdivided according to parameter thresholds in receiver operating characteristic curves and compared to TACE+Sorafenib group survival. The Gabor-1-90 (filter 0 ) was most significantly correlated with TTP. The TACE group was accordingly divided into the TACE-1 (Gabor-1-90 $\leq 3.6190$ ) and TACE-2 (Gabor-1-90 > 3.6190) subgroups; TTP was similar in the TACE-1 subgroup and TACE+Sorafenib group, but shorter in the TACE-2 subgroup. Only wavelet-3-D (filter 1.0) correlated with overall survival (OS), and was used for subgrouping. The TACE-5 (wavelet-3-D $\leq 12.2620$ ) subgroup and the TACE+Sorafenib group showed similar OS, while the TACE-6 (wavelet-3-D > 12.2620) subgroup had shorter OS. Gabor-1-90 and wavelet-3-D were consistent.Independent of tumor number or size, $\mathrm{CT}$ textural parameters are correlated with TTP and OS. Patients with lower Gabor-1-90 (filter 0) and wavelet-3-D (filter 1.0) should be treated with TACE and sorafenib. Texture analysis holds promise for appropriate selection of HCCs for this combination therapy.

\section{INTRODUCTION}

Hepatocellular carcinoma (HCC) has been proven to be a leading cause of cancer-related death worldwide [1-3]. More than half of HCCs have been diagnosed in China, and according to the most well-recognized Barcelona Clinic Liver Cancer (BCLC) stage, more than $75 \%$ patients are in stage $\mathrm{B}$ or $\mathrm{C}[4,5]$. According to the guidelines adopted by the European Association for the Study of the Liver/European Organization for Research and Treatment of Cancer (EASL-EORTC), American Association for the Study of Liver Diseases (AASLD), and National Comprehensive Cancer Network (NCCN), transcatheter arterial chemoembolization (TACE) is a fundamental therapy for these HCCs [4-6], while the combination of TACE and sorafenib has been considered to be promising 
[7-10], as sorafenib may control the elevation of vascular endothelial growth factor (VEGF) caused by TACE [11].

Although the combination of sorafenib and TACE was anticipated to be a breakthrough, and some studies had proven the safety and potential efficacy of the combination [7, 12], 2 recent randomized control trails drew a contrary conclusion about this combination therapy $[13,14]$. These controversies indicated that not all patients obtain a survival benefit from the combination. In addition, in some cases, TACE might be disturbed because of adverse effects (AEs) caused by sorafenib. Thus, a method allowing accurate patient selection for application of this combination is necessary. Multiples studies have sought potential factors, including clinical factors [15], biomarkers [16], and radiological characteristics [17], that could predict the efficacy of TACE or sorafenib. Although these studies provided meaningful insight in HCC prognosis, they fell short of accurate patients' identification, particularly for the combination of TACE and sorafenib.

In recent years, radiomics has become another significant field, in addition to genomics and proteomics, in oncology [18]. As a technique that categorizes regions of interest in an image by spatial variations in pixel intensities [19], texture analysis has been widely applied in a number of different cancers [20-26]. In studies of liver diseases, texture analysis has been used for prediction of postoperative hepatic insufficiency [27] and fibrosis assessment [28]. Furthermore, in a previous study, we have proven that texture analysis was promising for HCC patient stratification for determining the suitability of liver resection vs. TACE [29]. Therefore, we considered that it might be a potential method for selecting patients for combination therapy.

To prove our hypothesis, we conducted this study in 2 steps: firstly, we tested whether texture analysis could be prognostic in HCC patients treated with TACE; secondly, we verified whether the identified textural parameters could be used in selecting patients that may be suitable for combination therapy.

\section{RESULTS}

\section{Patients}

Two-hundred-and-sixty-one patients were included, of which 197 were treated with TACE and 64 were treated with TACE plus sorafenib (TACE+Sorafenib). By the enddate, 150 patients in the TACE group and 58 of $64(91 \%)$ patients in the TACE+Sorafenib had died. Fifteen of 197 $(8 \%)$ patients in the TACE group were lost to follow-up of overall survival. Furthermore, 191 of 197 (97\%) patients in the TACE group and 61 of $64(95 \%)$ in the TACE+Sorafenib group had recorded PD. During treatment, there were 74 $(37.6 \%)$ in the TACE group vs. $17(26.6 \%)$ patients in the TACE+Sorafenib group received ablation $(P=0.149)$. Their demographic were shown in Table.1.

\section{Screening of candidate textural parameters by Cox regression analysis}

For TTP, univariate Cox regression showed that 17 parameters in the Gabor filter ( 2 in filter 0,5 in filter 1.0 , and 10 in filter 1.5), and 16 wavelet transform ( 1 in filter 0,6 in filter 1.0, and 9 in filter 1.5) had $P$ values $<0.10$ (Supplementary Table E1). Among candidate clinical factors, only VI/EM had a $P$ value $<0.10$. Six separated multivariate Cox regression analyses, including both clinical factors and textural parameters, showed that Gabor-1-90 (filter 0), Gabor-1-135 (filter 1.0), Gabor-1-135 (filter 1.5), wavelet-2-D (filter 1.0) and wavelet-3-D (filter 1.5) were significantly related to TTP. In addition, VI/EM was also related to TTP in the Cox regression analysis for wavelet transform in filter 1.0 (Supplementary Tables S2, S3).

For OS, during follow-up, 16 patients were lost to follow-up after PD; hence, OS analysis was performed using the remaining 245 patients. In this analysis, univariate Cox regression analysis showed that 17 parameters in 5 Gabor filter (all in filter 1.0) and 2 wavelet transform (all in filter 1.0) had $P$ values $<0.10$ (Supplementary Table S1). Among candidate clinical factors, only sex had a $P$ value $<0.10$. Therefore, only 2 individual multivariate Cox regression analyses that included both clinical factors and textural parameters were performed; the results showed that sex and wavelet-3-D (filter 1.0) were significantly related to OS (Supplementary Tables S2, S3).

\section{ROC curves}

Among the selected textural parameters identified by multivariate Cox regression analyses, ROC curves were drawn to identify the thresholds. The results showed that the thresholds were 3.6190 for Gabor-1-90 (filter 0), 1.3995 for Gabor-1-135 (filter1.0), 0.5175 for Gabor-1-135 (filter 1.5), 18.3585 for wavelet-2-D (filter 1.0), 6.7515 for wavelet-2-D (filter 1.5), and 12.2620 for wavelet-3-D (filter 1.0), respectively.

\section{Kaplan-Meier analysis of disease progression in TACE patients}

For TTP, when the TACE group was separated by the threshold of Gabor-1-90 (filter 0), the 2 subgroups were statistically significantly different $(P<0.001$, Figure 1A). Similar results could be achieved by using Gabor-1-135 (filter 1.0), Gabor-1-135 (filter 1.5), wavelet2-D (filter 1.0), and wavelet-2-D (filter 1.5) (Figure 1B, 1C, 1D, and 1E, Supplementary Table S4). For OS, when the TACE group was separated by the threshold of wavelet-3-D (filter 1.0), the 2 subgroups were also statistically significantly different $(P<0.001$, Figure $1 \mathrm{~F}$, 
Supplementary Table S4).

\section{Kaplan-Meier analysis in all the patients}

Without detailed grouping, the TACE and TACE+Sorafenib groups showed no statistically significant difference in either TTP or OS (Figure 2A, 2G, Supplementary Table S4). This seemed to indicate that the combination of TACE and sorafenib had limited efficacy. However, when the TACE group were separated by the identified textural parameters, different results were found, as detailed below.

For TTP, when the TACE group was divided by Gabor-1-90 (filter 0) into TACE-1 (Gabor-1-90 $\leq 3.6190)$ and TACE-2 (Gabor-1-90 > 3.6190) subgroups, the difference between the 3 groups (TACE-1, TACE2 , and TACE + Sorafenib) were statistically significantly different $(P<0.001)$, arising from the difference between TACE- 2 and TACE+Sorafenib, but not between TACE- 1 and TACE+Sorafenib (Figure 2B). Similar results were achieved by wavelet-2-D (filter 1.5) (Figure 2F, Supplementary Table S4). When TACE was separated by the threshold of Gabor-1-135 (filter 1.0) into TACE-3 (Gabor-1-135 $\leq$ 1.3995) and TACE-4 (Gabor-1-135 $>1.3995$ ), statistically significant differences were found in all the pairwise comparisons (Figure 2C). Similar results were found when using Gabor-1-135 (filter 1.5) or wavelet2-D (filter 1.0) (Figures 2D, 2E, Supplementary Table S4).

For OS, when the TACE group was separated by wavelet-3-D (filter 1.0) into TACE-5 (wavelet-3-D $<12.2620$ ) and TACE-6 (wavelet-3-D > 12.2620), the difference between the 3 groups was statistically significant $(P<0.001)$. In pairwise comparisons, there was a statistically significant difference between TACE- 6 and TACE+Sorafenib, but not between TACE-5 and TACE+Sorafenib (Figure 2H, Supplementary Table S4).

\section{Cox regression analysis for all the patients}

For TTP, based on the results of Kaplan-Meier analysis of the data from all the patients, when separated by Gabor-1-135 (filter 1.0), Gabor-1-135 (filter 1.5), or wavelet-2-D (filter 1.0), some patients with lower values may still obtain a survival benefit from the combination of TACE and sorafenib. Thus, these parameters were inferior to the Gabor-1-90 (filter 0) and wavelet-2-D (filter 1.5) in identification of the most patients that would be suitable for combination therapy. Thus, we performed the Cox regression analysis as follows.

For TTP, univariate Cox regression analysis showed that, among the candidate clinical variables and subgrouping methods, only the subgrouping methods had a $P$ value $\leq 0.10$. Then, multivariate Cox regression analyses showed that subgrouping by Gabor-1-90 (filter 0 ) was statistically associated with TTP $(P=0.007)$, while subgrouping by wavelet-2-D (filter 1.5 ) identified no factor related to TTP (Table 2).

For OS, univariate Cox regression analysis showed that subgrouping by wavelet-3-D (filter 1.0) was the only factor with a $P$ value $\leq 0.10$. This also remained statistically significant in multivariate Cox regression
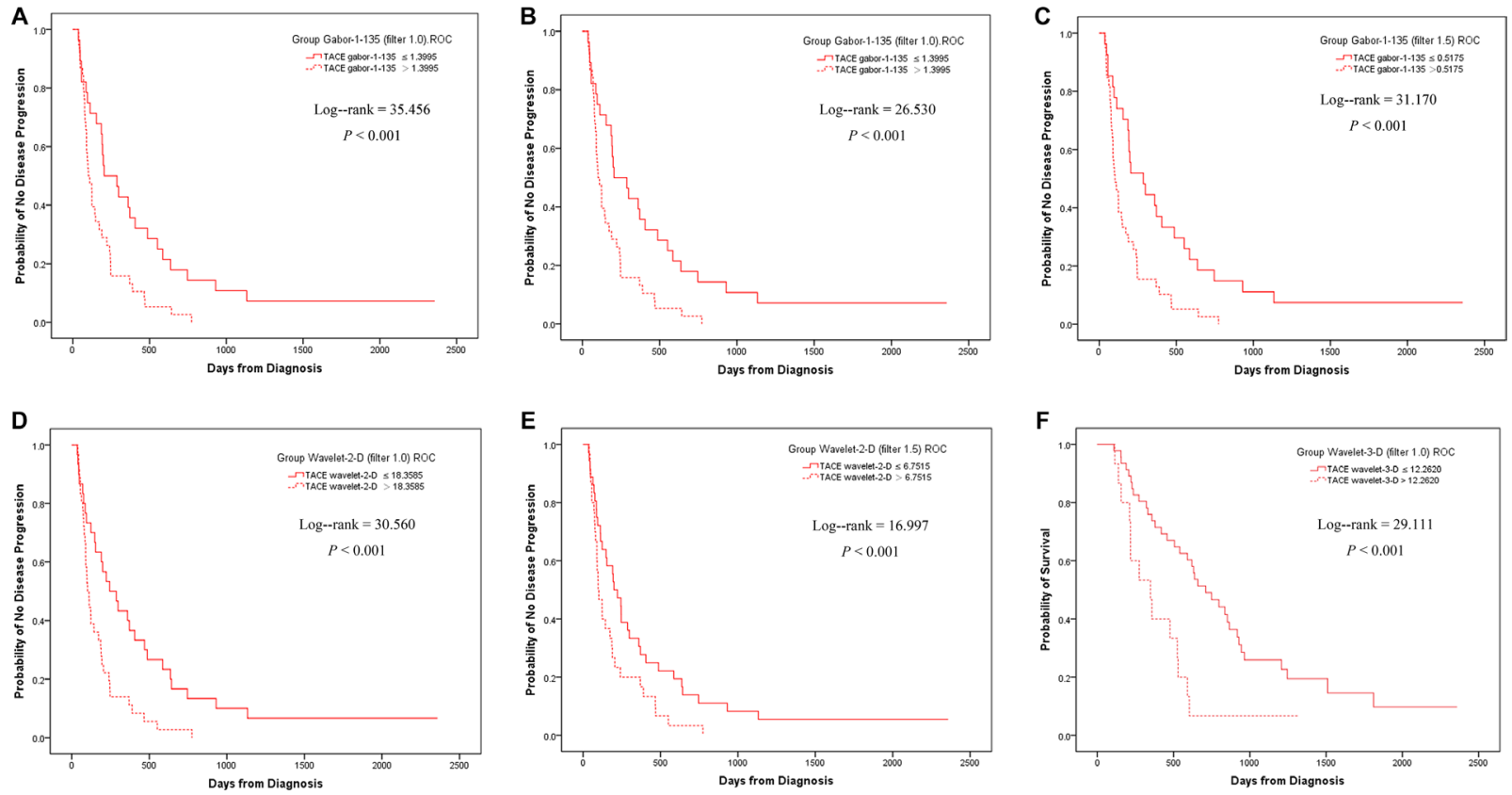

Figure 1: Kaplan-Meier analyses for TTP in TACE patients. When separated by the ROC threshold of Gabor-1-90 at filter 0, the two subgroups had a statistical difference in TTP (A). Similar results could be achieved by the threshold of Gabor-1-135 at filter 1.0 (B), Gabor-1-135 at filter 1.5 (C) wavelet-2-D at filter 1.0 (D) and wavelet-2-D at filter 1.5 (E). When separated by the threshold of wavelet-3-D at filter 1.0, the two subgroups had a statistical difference in OS (F). 
analysis $(P=0.013$; Table 2$)$.

\section{Further validation for the consistence between endpoints}

According to the results of Kaplan-Meier analysis in all the patients, Gabor-1-90 (filter 0) and wavelet-2-D (filter $1.5)$ performed best in identifying all the patients possibly suitable for the combination therapy. However, since wavelet-2-D (filter 1.5) did not have a statistical difference in Cox regression, Gabor-1-90 (filter 0) was superior.

Since Gabor-1-90 (filter 0) data were normally distributed between TACE-5 and TACE-6, independent $t$-tests were used for further analysis. The results showed that Gabor-1-90 was lower in the TACE-5 than in the TACE-6 group $(2.9512 \pm 0.8190$ vs. $3.5318 \pm 0.6609$,
$P=0.012)$, which confirmed the consistency between Gabor-1-90 (filter 0) and wavelet-3-D (filter 1.0).

\section{DISCUSSION}

Although studies have proven that sorafenib could suppress the elevation of VEGF after TACE and may bring a survival benefit to these patients [30, 31], this issue was still controversial [13, 14]. Furthermore, since sorafenib could increase side effects [9, 10, 13], sorafenib administration may influence liver function and disturb the schedule of TACE. Therefore, before using such combination therapy, patients should be selected to identify those who may truly experience a survival benefit.
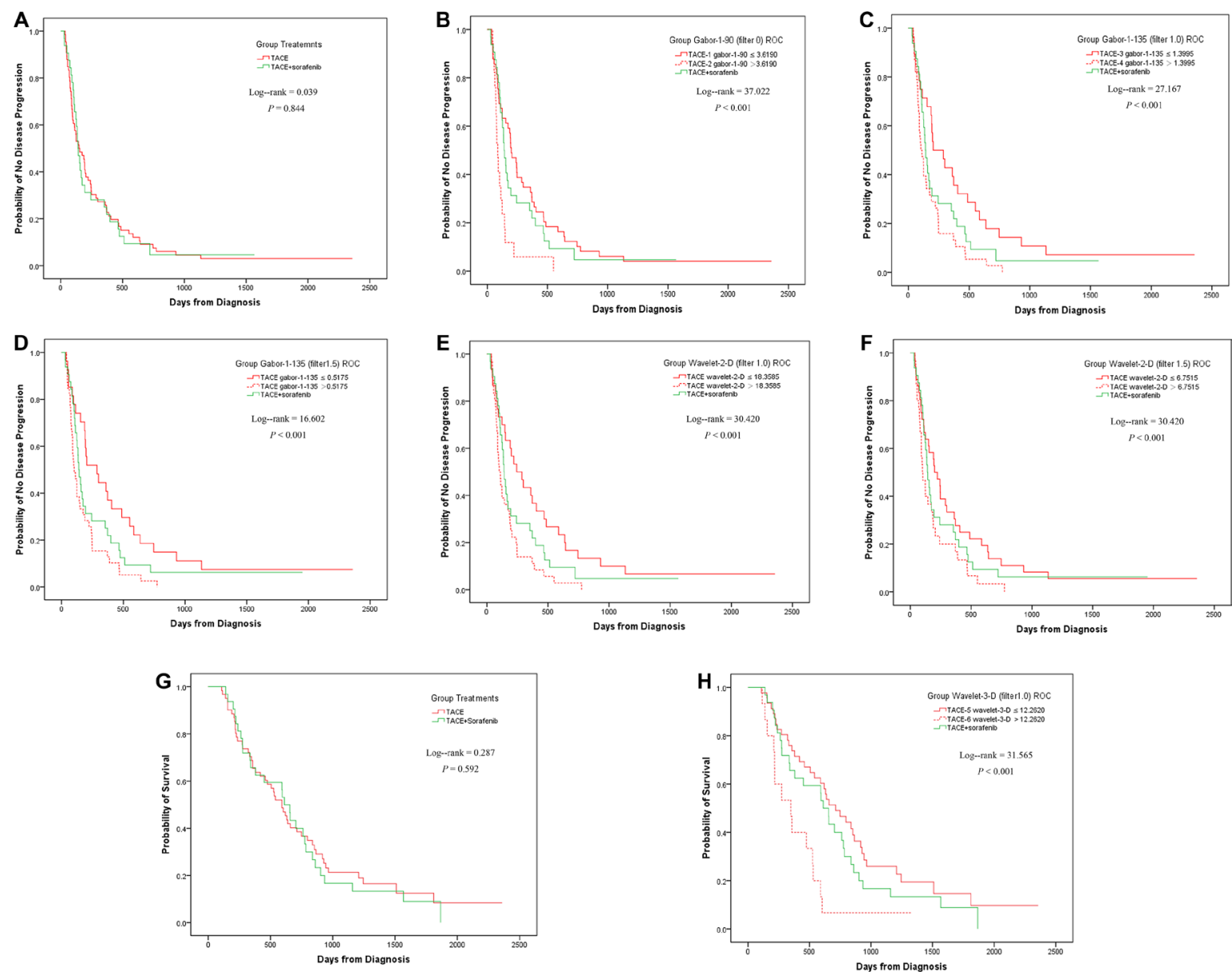

Figure 2: Kaplan-Meier analyses in all the patients. Without subgrouping, the TACE and TACE+Sorafenib group did not had a statistical difference in TTP (A). When the TACE group was divided by Gabor-1-90 at filter 0 into TACE-1 and TACE-2 subgroups, the difference between the 3 groups were statistically significantly different, arising from the difference between TACE-2 and TACE+Sorafenib, but not between TACE-1 and TACE+Sorafenib (B). Similar results were achieved by wavelet-2-D at filter 1.5 (F). When TACE was separated by the threshold of Gabor-1-135 at filter 1.0 into TACE-3 and TACE-4, statistically significant differences were found in all the pairwise comparisons (C). Similar results were found when using Gabor-1-135 at filter 1.5 (D) or wavelet-2-D at filter 1.0 (E). Without subgrouping, the TACE and TACE+Sorafenib group did not had a statistical difference in OS (G). When the TACE group was separated by wavelet-3-D at filter 1.0 into TACE-5 and TACE-6, the difference between the 3 groups was statistically significant. In pairwise comparisons, there was a statistically significant difference between TACE-6 and TACE+Sorafenib, but not between TACE-5 and TACE+Sorafenib (H). 
Table 1: Demographic and baseline characteristics of the patients

\begin{tabular}{|c|c|c|c|c|}
\hline & All $(N=261)$ & TACE $(N=197)$ & Sorafenib $(N=64)$ & $P$ \\
\hline Age & $56(20-83)^{*}$ & $58(20-84)^{*}$ & $54(20-79) *$ & 0.103 \\
\hline $\operatorname{Sex}(N)$ & & & & 0.094 \\
\hline Male & 241 & 185 & 56 & \\
\hline Female & 20 & 12 & 8 & \\
\hline BMI $\left(\mathrm{kg} / \mathrm{m}^{2}\right)$ & $24(15-32)^{*}$ & $24(15-32)^{*}$ & $23(16-33)^{*}$ & 0.840 \\
\hline Cause of disease (N) & & & & 1.000 \\
\hline $\mathrm{HBV}$ & 195 & 147 & 48 & \\
\hline $\mathrm{HCV}$ & 4 & 3 & 1 & \\
\hline Negative & 62 & 47 & 15 & \\
\hline Child-Pugh class (N) & & & & 0.231 \\
\hline A & 180 & 132 & 48 & \\
\hline $\mathrm{B}$ & 81 & 65 & 16 & \\
\hline \multicolumn{5}{|l|}{ BCLC (N) } \\
\hline $\mathrm{AB}$ & & 62 & 13 & 0.081 \\
\hline $\mathrm{B}$ & & 75 & 26 & \\
\hline $\mathrm{C}$ & & 60 & 25 & \\
\hline Vascular invasion & & & & 0.114 \\
\hline No & 176 & 138 & 38 & \\
\hline Yes & 85 & 59 & 26 & \\
\hline Cirrhosis & & & & 0.984 \\
\hline Yes & 196 & 148 & 48 & \\
\hline No & 65 & 49 & 16 & \\
\hline MD (mm) & 75 (42-187)* & $74(42-187)^{*}$ & $77(48-175)^{*}$ & 0.821 \\
\hline Lesion number $(\mathrm{N})$ & & & & 0.446 \\
\hline $\mathrm{N}=1$ & 113 & 93 & 20 & \\
\hline $\mathrm{N}=2$ & 67 & 33 & 34 & \\
\hline$N=3$ & 13 & 3 & 10 & \\
\hline $\mathrm{N} \geq 4$ & 68 & 68 & 0 & \\
\hline Albumin (g/L) & $35(21-48)^{*}$ & $35(22-44) *$ & $34(21-48)^{*}$ & 0.568 \\
\hline TBIL ( $\mu \mathrm{mol} / \mathrm{L})$ & $20(5-52)^{*}$ & $20(8-52)^{*}$ & $22(5-37)^{*}$ & 0.812 \\
\hline Prothrombin time & $14(12-16)^{*}$ & $14(12-15)^{*}$ & $14(12-16)^{*}$ & 0.418 \\
\hline ALT $(\mu \mathrm{mol} / \mathrm{L})$ & $38(10-566)^{*}$ & $38(15-566)^{*}$ & $39(10-236)^{*}$ & 0.480 \\
\hline $\operatorname{AFP}(\mathbf{N})$ & & & & 0.125 \\
\hline$<25 \mu \mathrm{g} / \mathrm{mL}$ & 69 & 57 & 12 & \\
\hline $25-400 \mu \mathrm{g} / \mathrm{mL}$ & 93 & 71 & 22 & \\
\hline$>400 \mu \mathrm{g} / \mathrm{mL}$ & 99 & 69 & 30 & \\
\hline
\end{tabular}

Abbreviations: * median (range) for data without normal distribution.

BCLC: Barcelona Clinic Liver Cancer; BMI: body mass index; HBV: hepatitis B virus; HCV: hepatitis C virus; MD: maximum diameter; TBIL: total bilirubin; ALT: alanine aminotransferase, AFP: alpha fetoprotein. 
Table 2: Multivariate cox regression for TTP and OS in all patients

\begin{tabular}{|c|c|c|c|}
\hline Cox model* & Factors & HR $(95 \%$ CI $)$ & $P$ \\
\hline \multicolumn{4}{|l|}{ TTP } \\
\hline \multirow[t]{3}{*}{ Gabor filter 0 Subgroups } & TACE+sorafenib & & 0.002 \\
\hline & Gabor- $1-90 \leq 3.6190$ & $0.802(0.505-1.274)$ & \\
\hline & Gabor-1-90 > 3.6190 & $2.184(1.190-4.007)$ & \\
\hline \multirow[t]{3}{*}{ Gabor filter 1. 0 Subgroups } & TACE+sorafenib & & 0.004 \\
\hline & Gabor-1-135 $\leq 1.3995$ & $0.455(0.231-0.898)$ & \\
\hline & Gabor-1-135 > 1.3995 & $1.298(0.827-2.036)$ & \\
\hline \multirow[t]{3}{*}{ Gabor filter 1.5 Subgroups } & TACE+sorafenib & & 0.008 \\
\hline & Gabor-1-135 $\leq 0.5175$ & $0.665(0.390-1.137)$ & \\
\hline & Gabor-1-135 > 0.5175 & $1.503(0.930-2.431)$ & \\
\hline \multirow{3}{*}{$\begin{array}{l}\text { Wavelet Transform filter } 1.0 \\
\text { Subgroups }\end{array}$} & TACE+sorafenib & & 0.007 \\
\hline & Wavelet-2-D $\leq 18.3585$ & $0.663(0.393-1.118)$ & \\
\hline & Wavelet-2-D $>18.3585$ & $1.486(0.912-2.422)$ & \\
\hline Wavelet Transform filter 1.5 & & None identified & \\
\hline \multicolumn{4}{|l|}{ OS } \\
\hline \multirow{3}{*}{$\begin{array}{l}\text { Wavelet Transform filter } 1.0 \\
\text { Subgroups }\end{array}$} & TACE + sorafenib & & 0.005 \\
\hline & Wavelet-3-D $<12.2620$ & $0.759(0.465-1.239)$ & \\
\hline & Wavelet-3-D > 12.2620 & $2.115(1.101-4.062)$ & \\
\hline
\end{tabular}

Abbreviations:"Seven separate multivariate cox regression analyses were performed: 6 for TTP and 1 for OS, and only variables with a statistical significance were listed.

TTP: time to progression; OS: Overall survival; TACE: transcatheter arterial chemoembolization; HR: hazard ratio; BCLC: Barcelona Clinic Liver Cancer; VI/EM: vascular invasion or extrahepatic metastasis.

Therefore, we conducted this study and proved that some textural parameters could assist in this selection process. Specifically, Gabor-1-90 (filter 0), Gabor-1-135 (filter 1.0), Gabor-1-135 (filter 1.5), wavelet-2-D (filter 1.0), and wavelet-2-D (filter 1.5) were indicative of TTP, while wavelet-2-D (filter 1.5) was indicative of OS.

In order to identify such patients appropriately, 3 steps were designed in this study. Firstly, textural parameters conveying prognostic information were identified and patients in the TACE group were divided into lower and higher subgroups according to their thresholds. TTP and OS in the 2 TACE subgroups were compared. Secondly, the TTP and OS of the subgroups were compared to those of the $\mathrm{TACE}+\mathrm{S}$ group. Multivariate Cox regression analysis was used to test whether the subgrouping represented independent prognostic factors. Thirdly, consistency was confirmed between the parameters of TTP and OS. After those steps, we proved that compared with the combination group, although some patients in the TACE group (e.g. TACE-1 and TACE-5) had similar survival, others (e.g. TACE-2 and TACE-6) indeed showed a shorter survival. Considering the comparability of baseline characteristics between the TACE + sorafenib group vs. the TACE-2 group (Gabor-1-90 > 3.6190) or TACE+sorafenib group vs. the TACE- 6 group (wavelet$3-\mathrm{D}>12.2620$ ), as well as the consistency of the first and second outcomes, we believed that if TACE-2 or TACE-6 were treated by the combination of TACE and sorafenib, they would probably have a better survival. Thus the cohort had similar textural features with TACE-2 or TACE-6 are the suitable patients for the combination therapy.

Although 5 parameters proved to be related to TTP, it is necessary to identify the best indicators in order to facilitate clinical application, and avoid contradiction by classification using different parameters. Based on the potential survival benefit afforded by sorafenib, we aimed to select the most appropriate patients. Therefore, when separated by Gabor-1-135 (filter 1.0), Gabor-1-135 (filter 1.5), or wavelet-2-D (filter 1.0), TTP still showed statistically significant differences between the lower value group (such as TACE-3) and the TACE+Sorafenib group. This indicated that some appropriate patients remained unidentified; these parameters were therefore inferior to Gabor-1-90 (filter 0) and wavelet-2-D (filter 1.5). In Cox 
regression analysis of the total patient group, subgrouping by wavelet-2-D (filter 1.5) did not result in statistically significant differences; therefore, Gabor-1-90 (filter 0) was the best parameter for determining benefit of TTP (Figure 3).

Since more confounders were induced after PD during treatment, OS was only used as the second endpoint. Following a similar process as for TTP, wavelet3-D (filter 1.0) was found to be the only parameter significantly related to OS. Nevertheless, since we used 2 different parameters for TTP and OS, we performed further validation and showed that the TTP endpoint by
Gabor-1-90 (filter 0) and the OS endpoint by wavelet-3-D (filter 1.0) were consistent (Figure 3).

In previous studies, radiomic on CT images has been suggested to be a potential prognostic biomarker. One seminal study identified that HCC imaging phenotypes, such as tumor margin scores, are strongly correlated with the gene expression program of the doxorubicin-response [32]. A thorough prospective radiomic analysis was performed in 1,019 patients with lung or head-and-neck cancer and showed that radiomics that included textural parameters could identify differences in the phenotypes

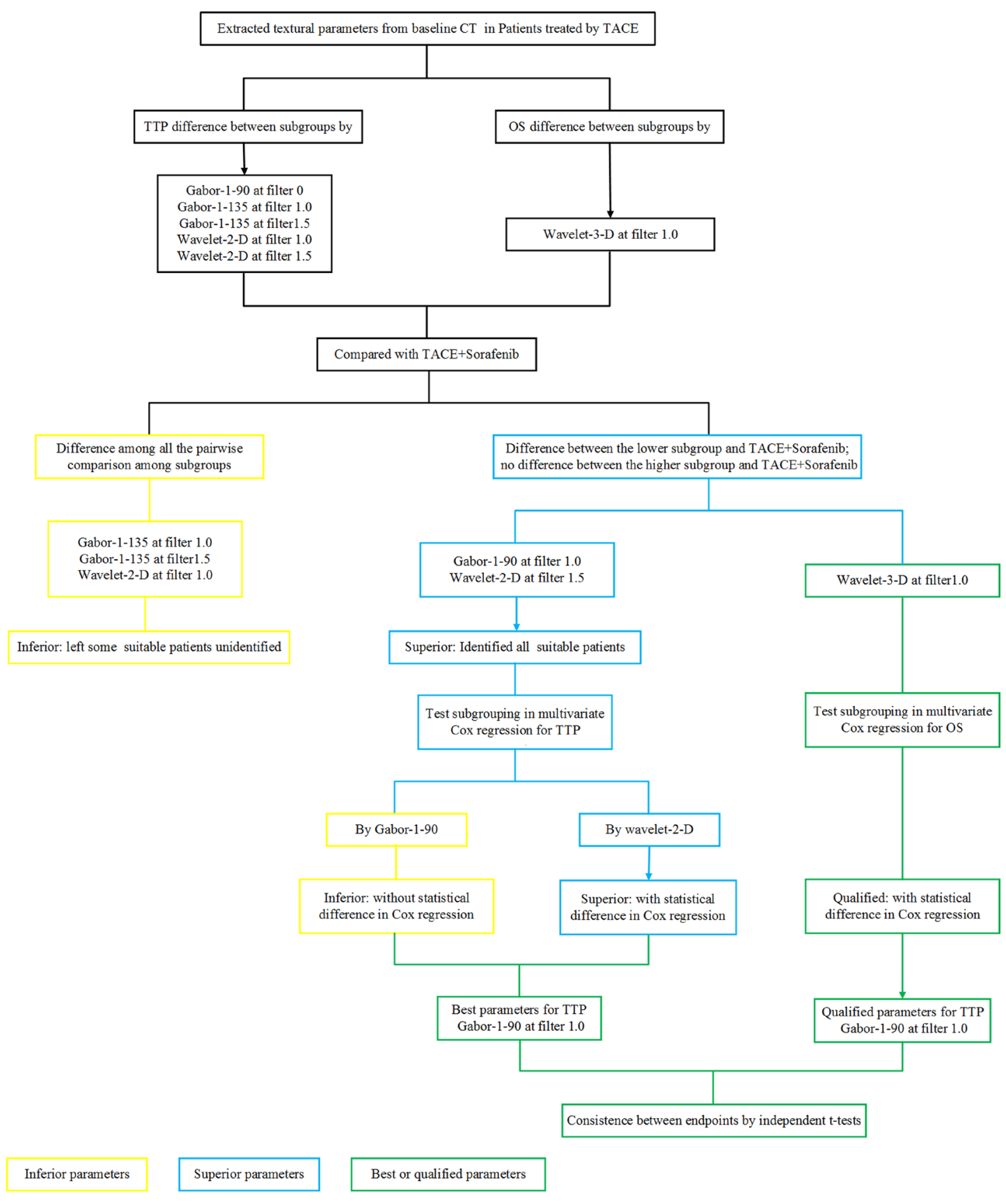

Figure 3: Flowchart of identifying the most suitable textural parameters. 
[33]. Additionally, studies on locally advanced lung adenocarcinoma identified significant corrections between CT textural parameters and distant metastasis [34]. Moreover, in primary colorectal cancer, texture features were also associated with the 5-year OS rate [35]. These studies highlighted the potential use of texture analysis in prognosis prediction, and we consider that it could facilitate personalized treatment.

The Gabor or wavelet transforms, with their spacefrequency decomposition abilities, have previously been applied in texture analysis. In the Gabor feature space, changes in object location, scale, and orientation are clearly detected [36]. The wavelet transform provides a natural adaptability to local signal properties and non-stationary signals, and thus can be used for texture characterization, segmentation, and classification [37, 38].

This study had some limitations. Firstly, as a retrospective study, possible confounding effects were present, although we attempted to control for these as far as possible; for this reason, we chose TTP rather than OS as our primary endpoint. And also theoretically, the incorporation of genomics and radiomics data may provide more comprehensive conclusion to this issue, but since biopsy was unnecessary in TACE, we could not achieve genomics data in most of the patients. Secondly, identification of patients for this combination therapy remains a highly complex matter. Therefore, the results of one study, performed at a single center, cannot be considered the final conclusion, particularly in terms of the threshold of the parameters identified. Thirdly, all regions of interest were manually drawn by the 2 radiologists; although excellent inter-observer agreement was reached, automatic segmentation would provide a more objective assessment and also save time. Fourthly, texture analysis has been performed for a limited tumor area, but not for the whole tumor; however, another study using only the largest tumor dimension have shown its promise as a predictive biomarker [21]. This could be addressed by improved software that would enable whole tumor segmentation and analysis.

In conclusion, textural parameters have proven to be promising in appropriately selecting patients for TACE and sorafenib combination treatment; in particular patients with Gabor-1-90 (filter 0) $\leq 3.8190$ or wavelet-2-D (filter $1.5) \leq 6.7515$ appear to be most likely to obtain survival benefit from the combination therapy.

\section{MATERIALS AND METHODS}

This retrospective study was approved by the Ethics Committee of Guangdong General Hospital. Informed consent was waived as all patient details were anonymized and de-identified prior to analysis.

\section{Patients}

Between September 2007 and December 2014, patients with HCC that was initially treated by TACE, with or without sorafenib, were enrolled from Guangdong General Hospital. The diagnosis of HCC was based on non-invasive criteria, according to the recommendation of EASL-EORTC and AASLD $[4,5]$. The inclusion criteria were as follows: (1) no previous treatment for HCC before initial TACE; (2) minimum follow-up of 3 years if still alive by the end of the study period (31 March 2015). Exclusion criteria were: (1) initially diagnosed based on magnetic resonance imaging (MRI) rather than computed tomography (CT); (2) irregular follow-ups; (3) accompanied by other cancers; (4) death unrelated to HCC; (5) incomplete CT data sets $(1.25$ $\mathrm{mm})$; (6) decreased liver attenuation values on precontrast $\mathrm{CT}$ images, steatosis on pre-contrast $\mathrm{CT}$ images. Supplementary Figure S1 summarizes the process for patients' inclusion and exclusion.

\section{Candidate clinical factors}

Candidate clinical factors for the Cox proportion hazard model included age, sex, maximum diameter (MD), lesion number (N: 1, 2, 3, and $>4$ ), cirrhosis (absence or presence), BCLC stage (AB, B, or C), Child-Pugh class (CP: A or B), vascular invasion or extrahepatic metastasis (VI/EM, absence or presence), alpha fetoprotein level (AFP, $<25 \mu \mathrm{g} / \mathrm{mL}, 25-400 \mu \mathrm{g} / \mathrm{mL}$, or $>400 \mu \mathrm{g} / \mathrm{mL}$ ), hepatitis infection (absence, A, B, C, D, or E).

Since biopsy is not essential for diagnosis and staging of HCC $[4,5]$, and as a gross specimen cannot be obtained during TACE, pathological-related data, such as TNM stage, were not available in this study. Moreover, we used the BCLC stage system instead, as recommended by the guidelines. In the BCLC staging system, considering the controversies involved in the staging of a single HCC $>5 \mathrm{~cm}$, we incorporated stage $\mathrm{AB}$ according to our own and others' previous studies [29, 39].

\section{Follow-ups}

The time interval between baseline $\mathrm{CT}$ and initial treatment was limited to less than 2 weeks. The followup interval was 4-8 weeks, including routine laboratory tests, chest radiography, and abdominal CT. Additional CT was routinely added when extrahepatic metastasis was suspected. Subsequent TACE and ablation were determined by our multidisciplinary team (MDT). In general, the decisions were made in reference to treatment response, evidence from current guidelines [4, 5], and the patients' status and intention to treatment. 


\section{Endpoints}

The primary endpoint was time to progression (TTP), which was defined as the time interval from diagnosis to disease progression (PD). PD was defined according to the mRECIST criteria [40]. The secondary endpoint was overall survival (OS), which was defined as the time interval from diagnosis to death. Considering the potential survival benefit of the combination therapy, we planned to identify the most suitable patients, either for TTP or for OS. Based on this, the textural parameters was tested for TTP and OS separately.

\section{CT technique}

All patients had undergone conventional contrastenhanced upper abdominal CT using the same scanner (LightSpeed VCT 64, GE Medical Systems, Waukesha, WI). The scan range was from the right diaphragmatic surface to the inferior border of the liver in the craniocaudal direction. After administering a non-ionic contrast medium, iopamidol $(370 \mathrm{mg}$ of iodine $/ \mathrm{mL}$; Iopamiro; Bracco, Milan, Italy) at a dose of $1.5 \mathrm{~mL} / \mathrm{kg}$ (maximum dose: $100 \mathrm{~mL}$ ) with a double-tube high-pressure syringe, at a rate of $3.5 \mathrm{~mL} / \mathrm{s}$, hepatic images were acquired at a fixed time point, with the portal venues phase (PVP) at a 70-s delay. Scan parameters were as follows: 120 $\mathrm{kV}$; automatic tube current modulation, $80-500 \mathrm{~mA}$; collimation: $64 \times 0.625$; noise index, 7 ; pitch/table speed, $0.984 / 39.37 \mathrm{~mm} / \mathrm{rot}$; rotation time, $0.6 \mathrm{~s}$; field of view, $300-450 \mathrm{~mm}$; matrix, $512 \mathrm{~mm}$. Image reconstruction was performed with a soft tissue kernel and a slice thickness of 1.25 and $5 \mathrm{~mm}$.

\section{Texture analysis}

For each pre-treatment examination, $1.25-\mathrm{mm}$ DICOM format axial portal venous phase images, through the largest cross-sectional area of tumor, were selected as the region of interest and transferred to 2 personal computers for texture analysis. Two radiologists (Reader 1 and Reader 2, with 5 and 4 years of experience in abdominal CT interpretation, respectively), who were blinded to patients' clinical and pathological information, reviewed the aforementioned images independently. The feature extraction methods of Gabor filter and wavelet analysis were used $[36,41]$. The process of texture analysis comprised 3 steps, as described in our previous paper, using MATLAB 2014a software (MathWorks Inc., Natick, $\mathrm{MA}$ ) and Image $\mathbf{J}$ software (National Institutes of Health, Bethesda, MD): (1) image filtration, (2) Gabor filter or wavelet transform (3) texture feature extraction [29].

\section{Statistical analysis}

Shapiro-Wilk test was applied for normality and Levene's test for homogeneity of variance. Differences in patient demographics and characteristics between groups were tested by using 2 independent-sample $t$-tests, MannWhitney $U$-tests, and chi-square tests.

The clinical characteristics of TACE patients were used in adjustment. Univariate Cox regression was used as a preliminary screening for variables to reduce the size of the feature set. Factors with a $P<0.10$ were entered into the subsequent multivariate Cox regression models (Forward: LR method). Textural parameters for each spatial filter were tested in separate Cox regression to assess the potential relationship between CT textural parameters of the primary mass and the TTP and OS respectively. Receiver operating characteristic curves (ROCs) were used to identify the thresholds. Finally, independent-sample $t$-tests or the Mann-Whitney $\mathrm{U}$ test, was performed to compare the identified textural parameters among the subgroups according to their normality and homogeneity.

All statistical analyses were performed with SPSS 20.0 (IBM SPSS Statistics, Armonk, NY) and R software (version 3.2.0; R Foundation for Statistical Computing, Vienna, Austria). A two-tailed $P$-value of less than 0.05 was considered statistically significant.

\section{ACKNOWLEDGMENTS}

We thank Shengli An, Southern Medical University, Department of Biostatistics, School of Public Health and Tropical Medicine for assistance with the statistical analysis.

\section{CONFLICTS OF INTEREST}

The authors have no conflicts of interest to declare.

\section{FINANCIAL SUPPORT}

This study was supported by supported by the National Natural Scientific Foundation of China (No. 81271654, No. U1301258 and No. 81571785), and Guangzhou Science and Technology Department, Industry Technology Research and Development Projects (No.201400000001-3).

\section{REFERENCES}

1. Njei B, Rotman Y, Ditah I, Lim JK. Emerging trends in hepatocellular carcinoma incidence and mortality. Hepatology. 2015; 61:191-199.

2. El-Serag HB. Epidemiology of viral hepatitis and hepatocellular carcinoma. Gastroenterology. 2012; 142:1264-1273.e1. 
3. Torre LA, Bray F, Siegel RL, Ferlay J, Lortet-Tieulent J, Jemal A. Global cancer statistics, 2012. CA Cancer J Clin. 2015; 65:87-108.

4. European Association for the Study of the Liver, European Organisation for Research and Treatment of Cancer. EASL-EORTC clinical practice guidelines: management of hepatocellular carcinoma. J Hepatol. 2012; 56:908-943.

5. Bruix J, Sherman M, American Association for the Study of Liver D. Management of hepatocellular carcinoma: an update. Hepatology. 2011; 53:1020-1022.

6. National Comprehensive Cancer Network. NCCN Clinical Practice Guidelines in Oncology: Antiemesis, V.1.2007. Available at http://www.nccn.org/professionals/physician gls/PDF/antiemesis.pdf. Accessed August 3, 2007.

7. Chao Y, Chung YH, Han G, Yoon JH, Yang J, Wang J, Shao GL, Kim BI, Lee TY. The combination of transcatheter arterial chemoembolization and sorafenib is well tolerated and effective in Asian patients with hepatocellular carcinoma: final results of the START trial. Int J Cancer. 2015; 136:1458-1467.

8. Park JW, Koh YH, Kim HB, Kim HY, An S, Choi JI, Woo SM, Nam BH. Phase II study of concurrent transarterial chemoembolization and sorafenib in patients with unresectable hepatocellular carcinoma. J Hepatol. 2012; 56:1336-1342.

9. Llovet JM, Ricci S, Mazzaferro V, Hilgard P, Gane E, Blanc JF, de Oliveira AC, Santoro A, Raoul JL, Forner A, Schwartz M, Porta C, Zeuzem S, et al. Sorafenib in advanced hepatocellular carcinoma. N Engl J Med. 2008; 359:378-390.

10. Cheng AL, Kang YK, Chen Z, Tsao CJ, Qin S, Kim JS, Luo R, Feng J, Ye S, Yang TS, Xu J, Sun Y, Liang H, et al. Efficacy and safety of sorafenib in patients in the asiapacific region with advanced hepatocellular carcinoma: a phase iii randomised, double-blind, placebo-controlled trial. Lancet oncol. 2009; 10:25-34.

11. Tsuchiya K, Asahina Y, Matsuda S, Muraoka M, Nakata T, Suzuki Y, Tamaki N, Yasui Y, Suzuki S, Hosokawa T, Nishimura T, Ueda K, Kuzuya T, et al. Changes in plasma vascular endothelial growth factor at 8 weeks after sorafenib administration as predictors of survival for advanced hepatocellular carcinoma. Cancer. 2014; 120:229-237.

12. Hu H, Duan Z, Long X, Hertzanu Y, Shi H, Liu S, Yang Z. Sorafenib combined with transarterial chemoembolization versus transarterial chemoembolization alone for advancedstage hepatocellular carcinoma: a propensity score matching study. PloS One. 2014; 9:e96620.

13. Lencioni R, Llovet JM, Han G, Tak WY, Yang J, Guglielmi A, Paik SW, Reig M, Kim DY, Chau GY, Luca A, Del Arbol LR, Leberre MA, et al. Sorafenib or placebo plus TACE with doxorubicin-eluting beads for intermediate stage HCC: The SPACE trial. J Hepatol. 2016.

14. Geschwind JF, Kudo M, Marrero JA, Venook AP, Chen XP, Bronowicki JP, Dagher L, Furuse J, Ladron de Guevara L, Papandreou C, Sanyal AJ, Takayama T, Ye SL, et al. TACE Treatment in Patients with Sorafenib-treated Unresectable
Hepatocellular Carcinoma in Clinical Practice: Final Analysis of GIDEON. Radiology. 2016; 279:630-40.

15. Sohn W, Paik YH, Cho JY, Lim HY, Ahn JM, Sinn DH, Gwak GY, Choi MS, Lee JH, Koh KC, Paik SW, Yoo BC. Sorafenib therapy for hepatocellular carcinoma with extrahepatic spread: treatment outcome and prognostic factors. J Hepatol. 2015; 62:1112-1121.

16. Llovet JM. Focal gains of VEGFA: candidate predictors of sorafenib response in hepatocellular carcinoma. Cancer cell. 2014; 25:560-562.

17. Fleckenstein FN, Schernthaner RE, Duran R, Sohn JH, Sahu S, Zhao Y, Hamm B, Gebauer B, Lin M, Geschwind JF, Chapiro J. 3D Quantitative tumour burden analysis in patients with hepatocellular carcinoma before TACE: comparing single-lesion vs. multi-lesion imaging biomarkers as predictors of patient survival. Eur Radiol. 2016.

18. Gillies RJ, Kinahan PE, Hricak H. Radiomics: Images Are More than Pictures, They Are Data. Radiology. 2016; 278:563-77. doi: 10.1148/radiol.2015151169.

19. Castellano G, Bonilha L, Li LM, Cendes F. Texture analysis of medical images. Clin Radiol. 2004; 59:1061-9.

20. Kassner A, Thornhill RE. Texture Analysis: A Review of Neurologic MR Imaging Applications. Am J Neuroradiol. 2010; 31:809-816.

21. Miles KA, Ganeshan B, Griffiths MR, Young RC, Chatwin CR. Colorectal cancer: texture analysis of portal phase hepatic CT images as a potential marker of survival. Radiology. 2009; 250:444-452.

22. Chappard C, Bousson V, Bergot C, Mitton D, Marchadier A, Moser T, Benhamou CL, Laredo JD. Prediction of femoral fracture load: cross-sectional study of texture analysis and geometric measurements on plain radiographs versus bone mineral density. Radiology. 2010; 255:536-543.

23. Chae HD, Park CM, Park SJ, Lee SM, Kim KG, Goo JM. Computerized texture analysis of persistent part-solid ground-glass nodules: differentiation of preinvasive lesions from invasive pulmonary adenocarcinomas. Radiology. 2014; 273:285-293.

24. Raman SP, Chen Y, Schroeder JL, Huang P, Fishman EK. CT texture analysis of renal masses: pilot study using random forest classification for prediction of pathology. Acad Radiol. 2014; 21:1587-1596.

25. Khalvati F, Wong A, Haider MA. Automated prostate cancer detection via comprehensive multi-parametric magnetic resonance imaging texture feature models. BMC Med Imaging. 2015; 15:27.

26. Waugh SA, Purdie CA, Jordan LB, Vinnicombe S, Lerski RA, Martin P, Thompson AM. Magnetic resonance imaging texture analysis classification of primary breast cancer. Eur Radiol. 2016; 26:322-330.

27. Simpson AL, Adams LB, Allen PJ, D'Angelica MI, DeMatteo RP, Fong Y, Kingham TP, Leung U, Miga MI, Parada EP, Jarnagin WR, Do RKG. Texture Analysis of Preoperative CT Images for prediction of postoperative hepatic insufficiency. J Am Coll Surgeons. 2015; 3:339-346. 
28. Daginawala N, Li B, Buch K, Yu H, Tischler B, Qureshi MM, Soto JA, Anderson S. Using texture analyses of contrast enhanced CT to assess hepatic fibrosis. Eur J Radiol. 2016; 85:511-7.

29. Li M, Fu S, Zhu Y, Liu Z, Chen S, Lu L, Liang C. Computed tomography texture analysis to facilitate therapeutic decision making in hepatocellular carcinoma. Oncotarget. 2016; 7:13248-13259. doi: 10.18632/oncotarget.7467.

30. Shim JH, Park JW, Kim JH, An M, Kong SY, Nam BH, Choi JI, Kim HB, Lee WJ, Kim CM. Association between increment of serum VEGF level and prognosis after transcatheter arterial chemoembolization in hepatocellular carcinoma patients. Cancer Sci. 2008.

31. Dufour JF, Hoppe H, Heim MH, Helbling B, Maurhofer O, Szucs-Farkas Z, Kickuth R, Borner M, Candinas D, Saar B. Continuous administration of sorafenib in combination with transarterial chemoembolization in patients with hepatocellular carcinoma: results of a phase I study. Oncologist. 2010; 15:1198-1204.

32. Kuo MD, Gollub J, Sirlin CB, Ooi C, Chen X. Radiogenomic analysis to identify imaging phenotypes associated with drug response gene expression programs in hepatocellular carcinoma. J Vasc Interv Radiol. 2007; 18:821-831.

33. Aerts HJ, Velazquez ER, Leijenaar RT, Parmar C, Grossmann P, Carvalho S, Bussink J, Monshouwer R, Haibe-Kains B, Rietveld D, Hoebers F, Rietbergen MM, Leemans CR, et al. Decoding tumour phenotype by noninvasive imaging using a quantitative radiomics approach. Nat Commun. 2014; 5:4006.

34. Coroller TP, Grossmann P, Hou Y, Rios Velazquez E, Leijenaar RT, Hermann G, Lambin P, Haibe-Kains B,
Mak RH, Aerts HJ. CT-based radiomic signature predicts distant metastasis in lung adenocarcinoma. Radiother Oncol. 2015; 114:345-350.

35. Ng F, Ganeshan B, Kozarski R, Miles KA, Goh V. Assessment of primary colorectal cancer heterogeneity by using whole-tumor texture analysis: contrast-enhanced CT texture as a biomarker of 5-year survival. Radiology. 2013; 266:177-84. doi: 10.1148/radiol.12120254.

36. Kamarainen JK, Kyrki V, Kalviainen H. Invariance properties of Gabor filter-based features-overview and applications. IEEE T Image Processing. 2006; 15:10881099.

37. Bharati MH, Liu JJ, MacGregor JF. Image texture analysis: methods and comparisons. Chemometr Intell Lab. 2004; 72:57-71.

38. Hackmack K, Paul F, Weygandt M, Allefeld C, Haynes JD, Alzheimer's Disease Neuroimaging I. Multi-scale classification of disease using structural MRI and wavelet transform. Neuroimage. 2012; 62:48-58.

39. Vitale A, Burra P, Frigo AC, Trevisani F, Farinati F, Spolverato G, Volk M, Giannini EG, Ciccarese F, Piscaglia F, Rapaccini GL, Di Marco M, Caturelli E, et al. Survival benefit of liver resection for patients with hepatocellular carcinoma across different Barcelona Clinic Liver Cancer stages: a multicentre study. J Hepatol. 2015; 62:617-624.

40. Lencioni R, Llovet JM. Modified RECIST (mRECIST) assessment for hepatocellular carcinoma. Semin Liver Dis. 2010; 30:52-60.

41. Mallat SG. A theory for multiresolution signal decomposition: the wavelet representation. IEEE Trans Pattern Anal Mach Intell. 1989; 11:674-693. 